\title{
Adding an extra condition: a general method to design double freeform-surface lens for LED uniform illumination
}

Run Hu and Xiaobing Luo*

\author{
* Correspondence: \\ luoxb@hust.edu.cn \\ School of Energy and Power \\ Engineering, Huazhong University \\ of Science and Technology, Wuhan \\ 430074, China
}

\begin{abstract}
Uniform illumination is an essential optical requirement for light-emitting diode (LED) applications. In this paper, a general method was proposed to design double freeform-surface lenses for uniform illumination of LED packages. Detailed algorithms of the design method were presented, in which the inner and outer surfaces of the lenses could be designed simultaneously. This problem can't be solved unless providing an extra condition. Two kinds of extra conditions were introduced to design the lens and validate the method. Besides uniform illumination, the present method can also realize extra functions with providing different extra conditions, like conformal phosphor coating and minimum Fresnel loss. The present method can be extended to design various freeform-surface lenses for LED uniform illumination provided that different conditions are added based on different application requirements.
\end{abstract}

Keywords: Lens; Light-emitting diode; Non-imaging optics; Illumination

\section{Background}

Recently, light-emitting diodes (LEDs) have become a vast lighting market due to its extraordinary features [1-5]. The direct output of a bare LED chip, however, is usually a circle spot with non-uniform illuminance distribution; therefore it can't be applied without packaging. Hence, appropriate primary or secondary optics are usually used to redistribute the spatial light distribution so that high quality LED illumination can be obtained by controlling the light pattern, illumination uniformity, etc. Among all the LED optics, a freeform lens has the advantages in abundant design degree of freedom (DoF), compact size and accurate light pattern control, thus it has become the dominant optics in LED illumination. There are many methods to design freeform lens, such as simultaneous multiple surface (SMS) method [6-8], partial differential equations method [9], tailored freeform surface method [10], discontinuous [11] or continuous [12-16] freeform lens method. These design methods except the SMS method, however, usually deal with the outer surface and the inner surface is considered as hemispherical shape for simplicity, which would cause light energy loss and abandon an important design freedom in the inner surface of freeform lenses. The SMS method is good at controlling the light direction precisely, but it is some difficult and lacks the ability to realize highly uniform illumination.

In this paper, we tried to develop a general method to design double freeformsurface lenses for LED uniform illumination. The algorithms of the design method

(c) $2014 \mathrm{Hu}$ and Luo; licensee Springer. This is an Open Access article distributed under the terms of the Creative Commons Attribution License (http://creativecommons.org/licenses/by/2.0), which permits unrestricted use, distribution, and reproduction in any medium, provided the original work is properly credited. 
were presented in detail. Two kinds of extra conditions were introduced to validate the method.

\section{Methods}

For a LED package, the light emanated from the LED light source is actually refracted twice when transmitting from the inner and the outer surfaces of the lens. As shown in Figure 1, the inner surface refracts the incident ray $\overrightarrow{O A}$ into first output ray $\overrightarrow{A B}$, which is the incident ray for the outer surface. The outer surface refracts the second incident ray $\overrightarrow{A B}$ into second output ray $\overrightarrow{B R}$, which irradiates at the corres-ponding point $R$ on the target plane. According to Snell's law, the rays $\overrightarrow{O A}, \overrightarrow{A B}, \overrightarrow{B R}$ must satisfy the following equations.

$$
\begin{aligned}
& n_{1} \frac{\overrightarrow{O A}}{|\overrightarrow{O A}|} \times \vec{N}_{1}=n_{2} \frac{\overrightarrow{A B}}{|\overrightarrow{A B}|} \times \vec{N}_{1} \\
& n_{2} \frac{\overrightarrow{A B}}{|\overrightarrow{A B}|} \times \vec{N}_{2}=n_{3} \frac{\overrightarrow{B R}}{|\overrightarrow{B R}|} \times \vec{N}_{2}
\end{aligned}
$$

where $\vec{N}_{1}$ and $\vec{N}_{2}$ are the normal vectors on the inner and outer surfaces, respectively. $n_{1}, n_{2}, n_{3}$ are the refractive indices of each area, respectively. $n_{2}$ is the refractive index of the lens material, and $n_{3}$ equals 1.00 because the corresponding area is air. When the lens is primary lens, $n_{1}$ is the refractive index of the silicone or phosphor silicone matrix; when the lens is secondary lens, $n_{1}$ equals 1.00 because the area is air.

From Eqs. (1)-(2), we may discover that these two equations can't be solved because the number of unknowns is more than the number of equations. But if we assume the inner surface as hemispherical surface, $\overrightarrow{O A}$ and $\overrightarrow{A B}$ are parallel and Eq. (1) can be neglected. This is the exact reason why most researchers simplify the problems by considering the inner surface as hemispherical. At the situation of this letter, we want to design the two surfaces simultaneously. We adopted the practical nonimaging optical design method to design the lens [12-16]. After the meshing of light source and target

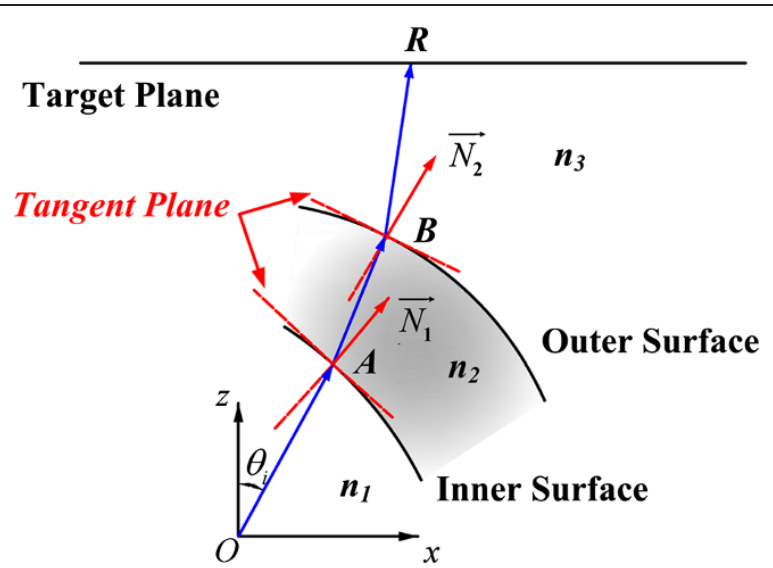

Figure 1 (Color online) Schematic of typical light path through double freeform surface lens. $\theta_{i}$ is the edge angle of incident ray. 
plane, we can obtain the vector of each incident ray (i.e. the edge angle $\theta_{i}$ ) and the coordinates of corresponding points on the target plane. Then, we supposed that the inner and outer surfaces can be described by surface functions $f_{1}(x, y, z)$ and $f_{2}(x, y, z)$, respectively. The tangent plane at each point is the differential coefficient of the functions $f_{1}(x, y, z)$ and $f_{2}(x, y, z)$ at each point, and the unit normal vector is the orthogonal of the tangent plane at each point. To make the indefinite equations solvable, we can add appropriate extra conditions to balance the number of unknowns and equations. There may be a lot of extra conditions, which can help solve the problem, like surface functions relationship control, light deviation control, etc. Different extra conditions correspond to different applications. Two kinds of extra conditions are illustrated and discussed below.

1) Surface function relationship control

As one kind of extra condition, we also can supply the relationship between the two surfaces. The relationship can be assigned based on appropriate assumptions. As the simplest cases, we can give the function of one surface and solve the other surface of the freeform-surface lens. For example, we can give the function of inner surface to calculate the function of the outer surface and vice versa.

As shown in Figure 2(a), when the function of the outer surface is given, i.e. $f_{2}(x, y, z)$ is known, we firstly fix a point $A_{0}$ as the vertex of the inner surface of the lens, and the normal vector at this point is vertical up. The second point $A_{1}$ can be calculated by the intersection of the incident ray $\overrightarrow{O A}_{1}$ and the tangent plane of the point $A_{0}$. Then applying Eq. (2), we can calculate the point $B_{1}$ on the outer surface since the unit normal vector at point $B_{1}$ is determined by the function $f_{2}(x, y, z)$ and the corresponding point $R_{1}$ is also determined previously. Then the unit normal vector at point $A_{1}$ is also calculated by applying Eq. (1) and the tangent plane at point $A_{1}$ is consequently determined. By repeating the this process until the edge angle $\theta_{i}$ equals $90^{\circ}$, we can get all the points and their unit normal vectors on the inner surface. As shown in Figure 2(b), when the function of the inner surface is given, i.e. $f_{1}(x, y, z)$ is known, we can calculate the outer surface with the same method. After obtaining all the coordinates on the inner or outer surface, we fit these points to form the contour line of the lens's cross Section.

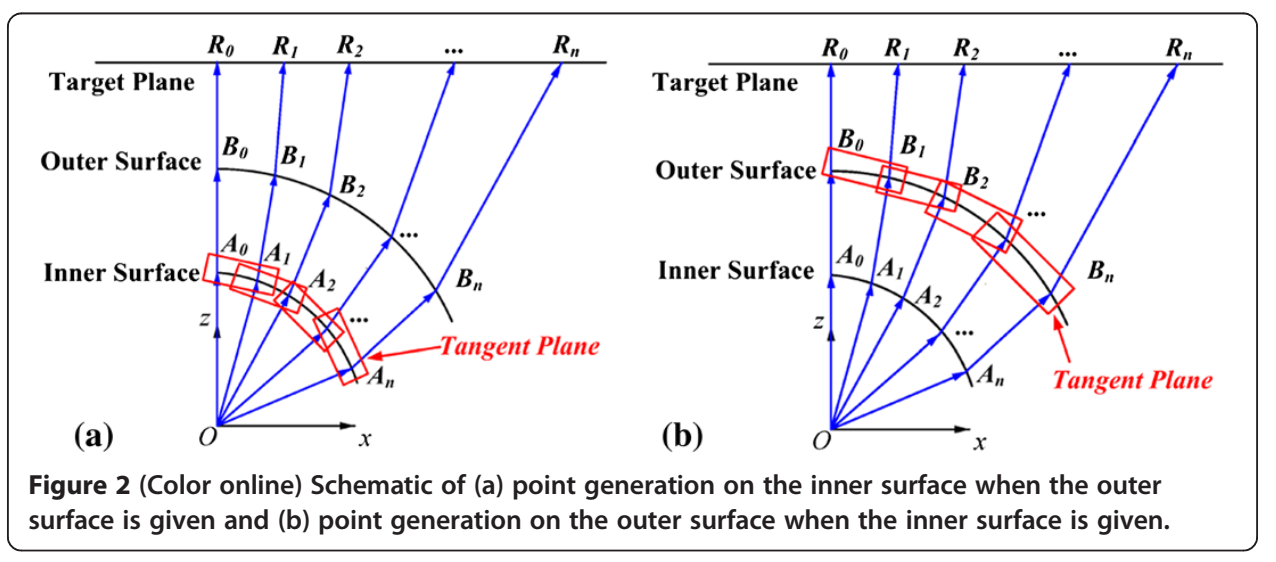


2) Light deviation control

As another kind of extra condition, we can control the two light deviation angles on the two surfaces with proper ratio. For each ray, the total light deviation angle from the incident ray $\overrightarrow{O A}$ to the output ray $\overrightarrow{B R}$ can be obtained. With proper ratio, we can divide the total deviation angle as two components, corresponding to the two refractions on the inner and outer surfaces respectively. As shown in Figure 3, we firstly fixed point $A_{O}$ and point $B_{O}$ as the vertex of the inner and outer surface of the lens, respectively. The normal vectors at these two points were vertical up. The second point $A_{1}$ on the inner surface could be calculated by the intersection of the incident ray $\overrightarrow{O A}_{1}$ and the tangent plane of the point $A_{0}$. With proper deviation angles, we could obtain the ray $\overrightarrow{A_{1} B_{1}}$ and ray $\overrightarrow{B_{1} R_{1}}$. The second point $B_{1}$ on the outer surface could be calculated by the intersection of the ray $\overrightarrow{A_{1} B_{1}}$ and the tangent plane of the point $B_{0}$. By applying Eqs. (1)-(2), we could calculate the unit normal vectors and the tangent planes at point $A_{1}$ and point $B_{1}$. And then we could obtain the third point $A_{2}$ on the inner surface by the intersection of the incident ray $\overrightarrow{O A}_{2}$ and the tangent plane of the point $A_{1}$. By repeating this process until the edge angle $\theta_{i}$ equaled $90^{\circ}$, we could get all the points and their unit normal vectors on the two surfaces. After obtaining all the coordinates on the inner and outer surfaces, we fit these points to form the contour line of the lens's cross section. If the lens is designed as axis-symmetrical lens, then we can get the freeform lens by rotating the contour line around the symmetry axis. If not, we can use the similar method to calculate the other contour lines. After obtaining all the contour lines, the lofting method can be used to form smooth surfaces of the lens between the contour lines [14].

What should be emphasized is that this general design method has many design DoFs. The extra conditions could be varied according to the different requirements and the resulting double freeform-surface lenses have different applications. Even for the two aforementioned extra conditions, it also has many design DoFs. As long as the optical performance, volume and profile of the designed lens are acceptable, the inner surface could be given as spherical, cubic, cylinder or even spheroidic and parabolic, while the outer surface is freeform, and vice versa. The ratio of the two deviation angle is also can be changed according to different applications. With

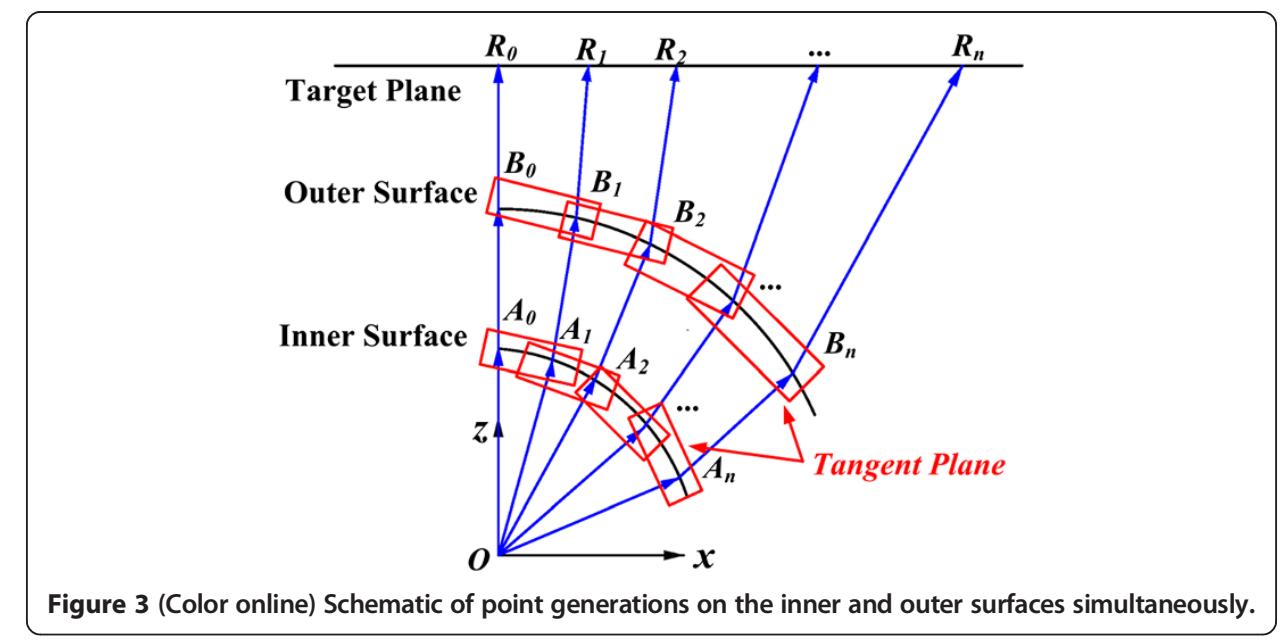


other extra conditions, the method could be extended to design different double freeform-surface lenses.

\section{Results and discussions}

To validate the above design method, we designed several examples by providing with different extra conditions. We also built the contrastive lenses where we replaced the freeform surfaces with hemispherical surfaces. Monte Carlo ray-tracing simulations were conducted to demonstrate the illumination performance. In all the simulations, the distance between the light source and the target plane was $50 \mathrm{~mm}$ and the radius of the target plane was $100 \mathrm{~mm}$. The material of the lenses were selected as polymethylmethacrylate (PMMA) whose refractive index is 1.4935 . To evaluate the illumination uniformity, the variation coefficient of root mean square error, or CV(RMSE) in short, was calculated. It is defined as [16-19]

$$
C V(R M S E)=R M S E / \bar{x}
$$

where the RMSE is the standard error and $\bar{x}$ is the mean value of the sample points of the target plane. The smaller the $C V(R M S E)$ is, the higher the uniformity is.

\section{Design example I}

The function of the inner surface of the freeform lens in the first design sample was given as

$$
f_{1}(x, y, z)=x^{2}+y^{2}-1=0, z \in[0,1]
$$

where the inner surface of the lens was a cylinder and the outer surface was freeform. The initial height of the outer surface of the lens was set as $2 \mathrm{~mm}$. With the present design method, we calculated the outer surface and built the freeform lens, as shown in Figure 4(a). The contrastive lens was illustrated in Figure 5(a).

The illumination performance on the target plane was shown in Figure 4(b) and its CV(RMSE) was 0.3967. For the contrastive case, the illumination performance was illustrated in Figure 5(b) and its $C V(R M S E)$ was as high as 3.2996. From the comparisons, we could see that the present freeform lens could enhance the illumination uniformity $87.98 \%$ and decrease the light loss. To figure out the reasons, we examined the light propagation path when transmitting across the lens. As shown in Figure 6, it was

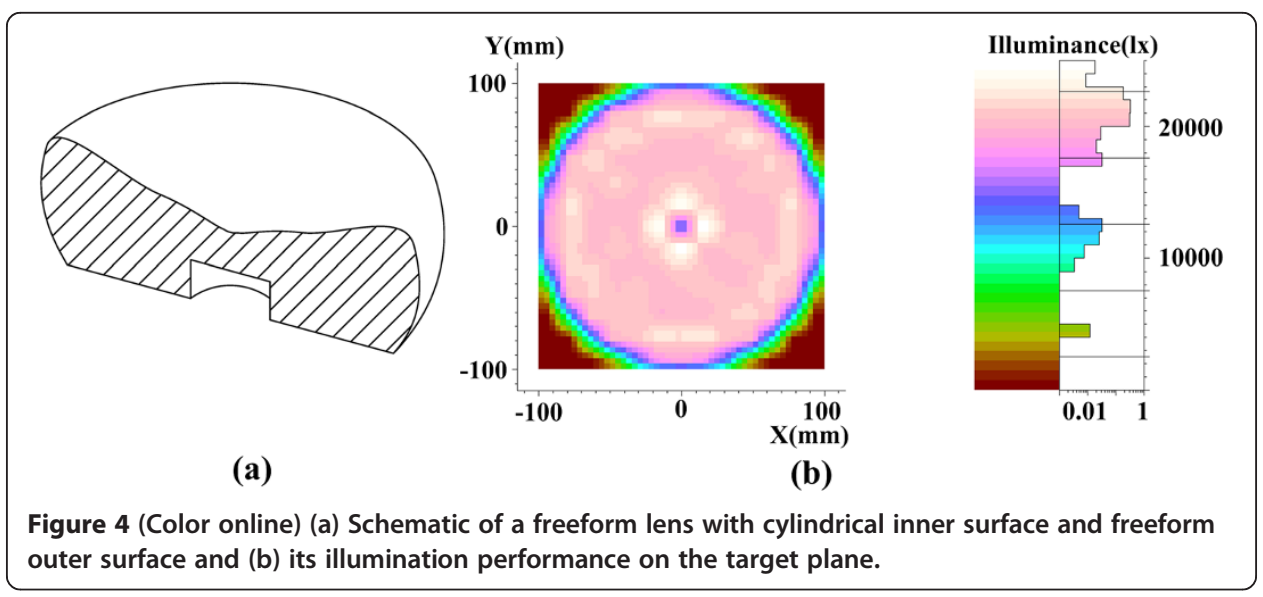




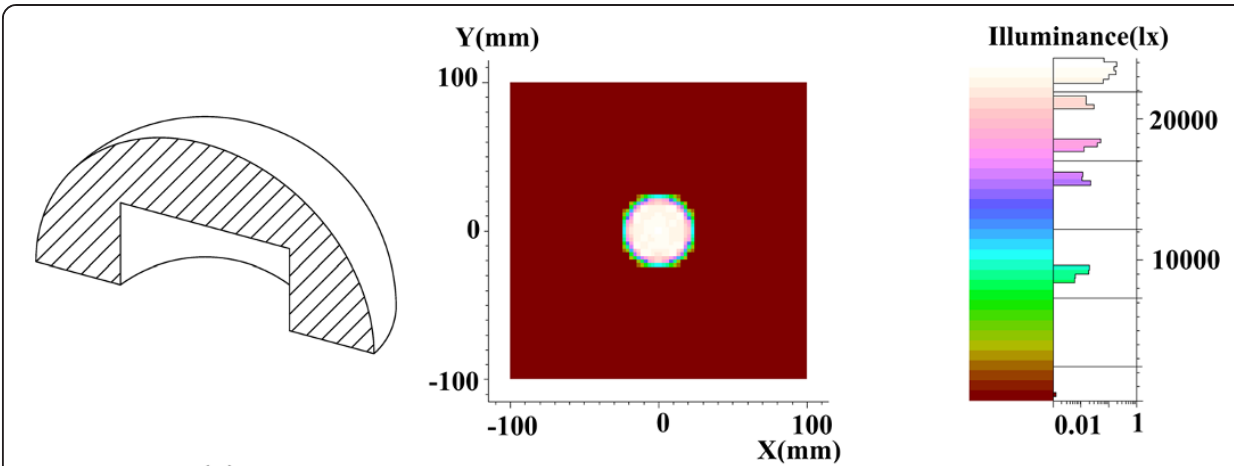

(a)

(b)

Figure 5 (Color online) (a) Schematic of contrastive lens with cylindrical inner surface and hemispherical outer surface and (b) its illumination performance on the target plane.

found that most light eradiated from the light source could reach the target plane when crossing the freeform lens, while only a small fraction of the light could reach the target plane when crossing the contrastive lens and most light were lost due to the refraction and total inner reflection. That's the exact reason of the light spot formation on the target plane in Figure 5(b). From the comparisons in Figures 4, 5 and 6, we can see that the freeform lens could increase the illumination uniformity and decrease the light loss.

Inspired by the flat inner surface of such double freeform lens, we designed a novel lens to realize uniform illumination and conformal phosphor coating simultaneously. The flat inner surface can realize uniform thickness of phosphor layer, and then we designed the outer surface to achieve uniform illumination by controlling the direction of the emergent ray from the preset inner surface. The detailed design process and validation can be referred to our previous study [17].

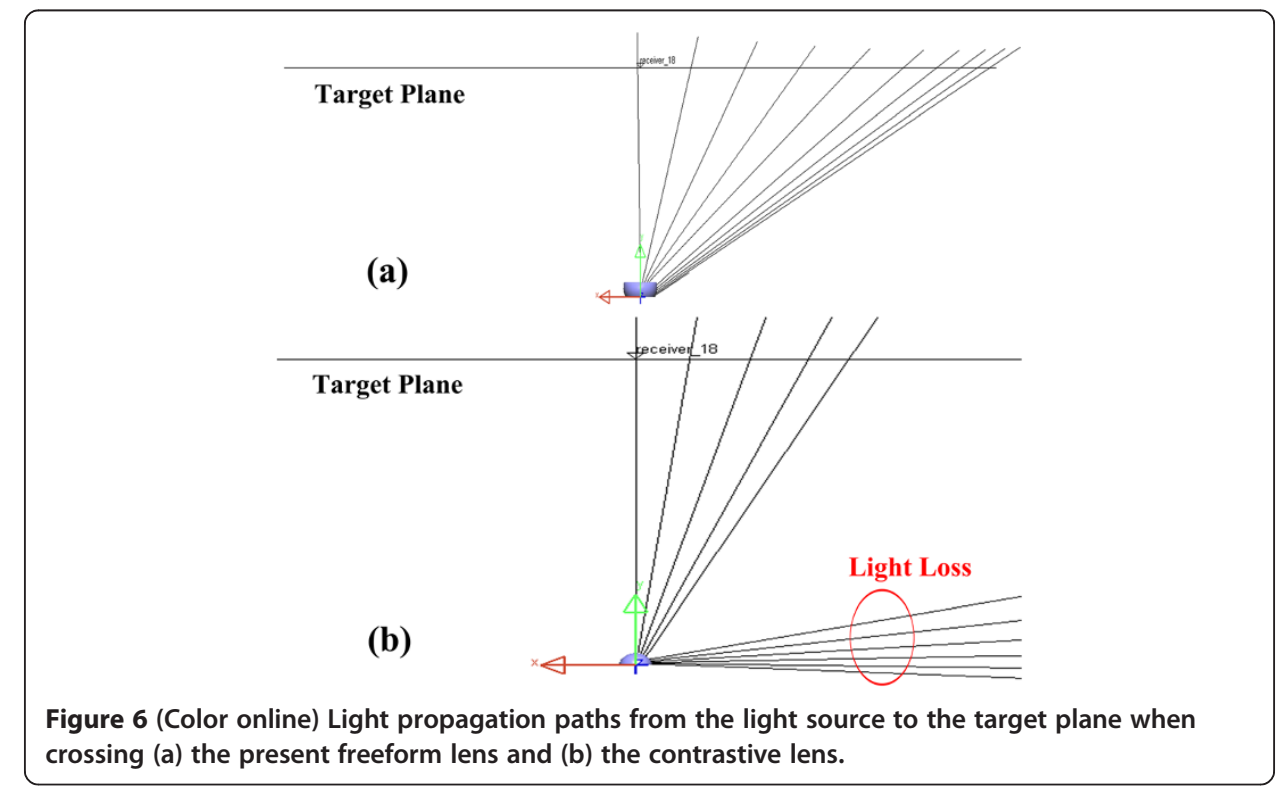




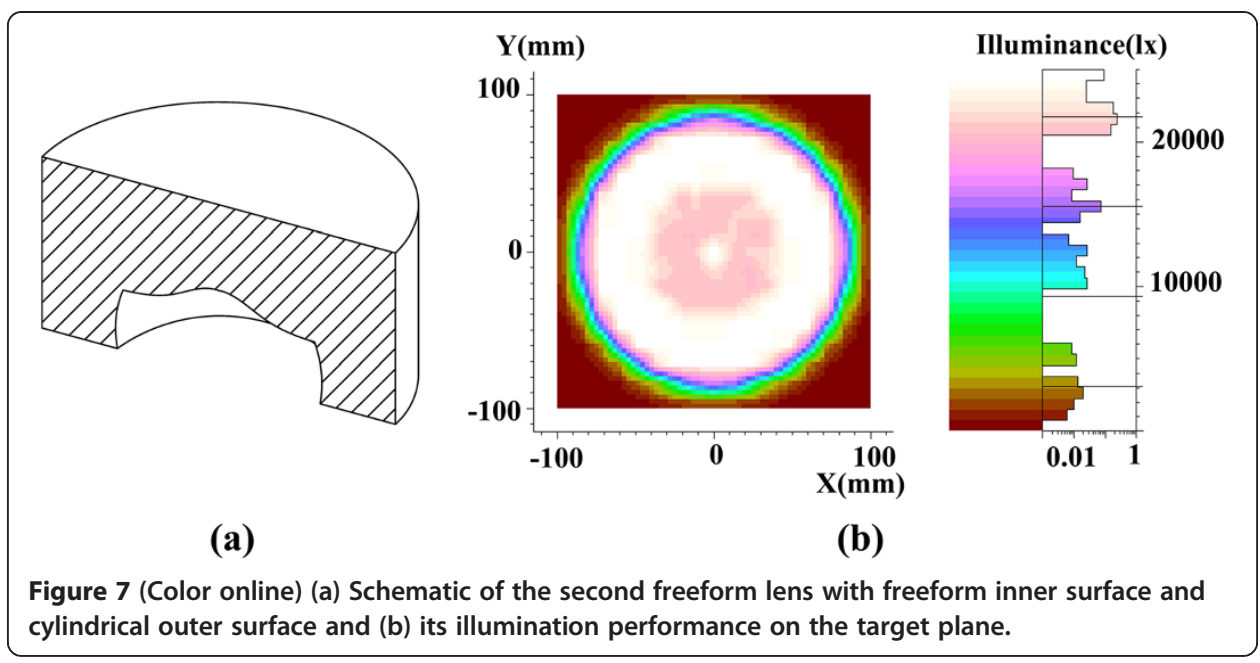

\section{Design example II}

In the second design example, the function of the outer surface of the freeform lens was given as

$$
f_{2}(x, y, z)=x^{2}+y^{2}-4=0, z \in[0,2]
$$

where the outer surface of the lens was a cylinder and the inner surface was freeform. The initial height of the inner surface of lens was set as $1 \mathrm{~mm}$. With the same method, we calculated the coordinates of inner freeform surface and built the freeform lens as shown in Figure 7(a). Figure 8(a) shows the contrastive lens whose inner surface was spherical and outer surface was cylindrical. Their illumination performances were illustrated in Figure 7(b) and Figure 8(b). The CV(RMSE) of the freeform lens was 0.7381, while the $C V(R M S E)$ of the contrastive lens was 1.266 . From the comparison, we could see that the illumination uniformity of the freeform lens designed by the present method was enhanced $41.70 \%$.

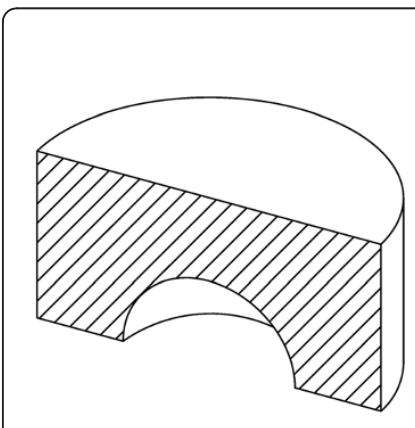

(a)
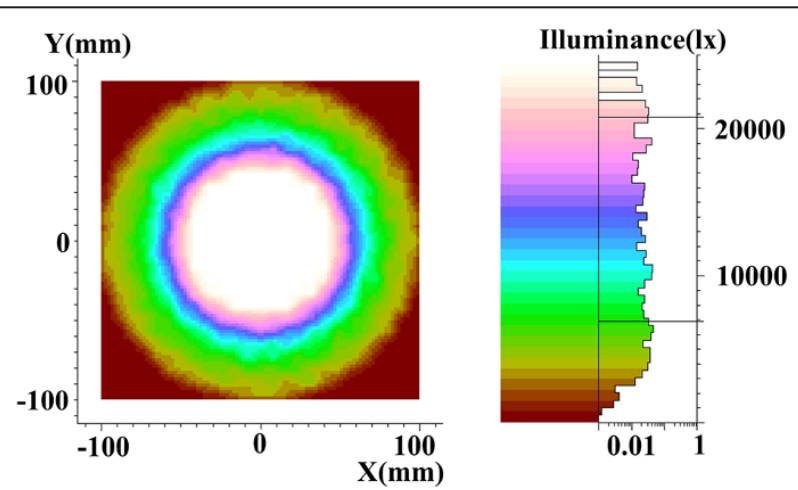

(b)

Figure 8 (Color online) (a) Schematic of the second contrastive lens with spherical inner surface and cylindrical outer surface and (b) its illumination performance on the target plane. 


\section{Design example III}

Figure 9 shows another kind of double freeform-surface lens, which was designed by controlling the deviation angle on the inner and outer surfaces $[18,19]$. We distributed the total deviation angle into two parts corresponding to the two refractions on the inner and outer surfaces of the lens. The incident ray deviates for the first time when transmitting through the inner surface, and then deviates for the second time when transmitting through the outer surface. With this method illustrated in Figure 3, nine cases were designed. Monte Carlo ray-tracing simulations and Fresnel loss calculations were conducted. It was proven that the present double freeform-surface lens can realize uniform illumination as well as minimum Fresnel loss.

\section{Conclusions}

In summary, a general method was developed to realize uniform illumination of LED packages by adding extra conditions. The algorithms of the design method were presented in detail. Two kinds of extra conditions were introduced to design the lens. It is found that the lens design by present method not only can realize uniform illumination, but also can realize another extra function as well, like phosphor conformal coating and minimum Fresnel loss. Different double freeform-surface lenses can be designed with different extra conditions. Since the extra conditions may be flexible according to the different requirements, the general design method may have plentiful design DoFs and extensive applications.

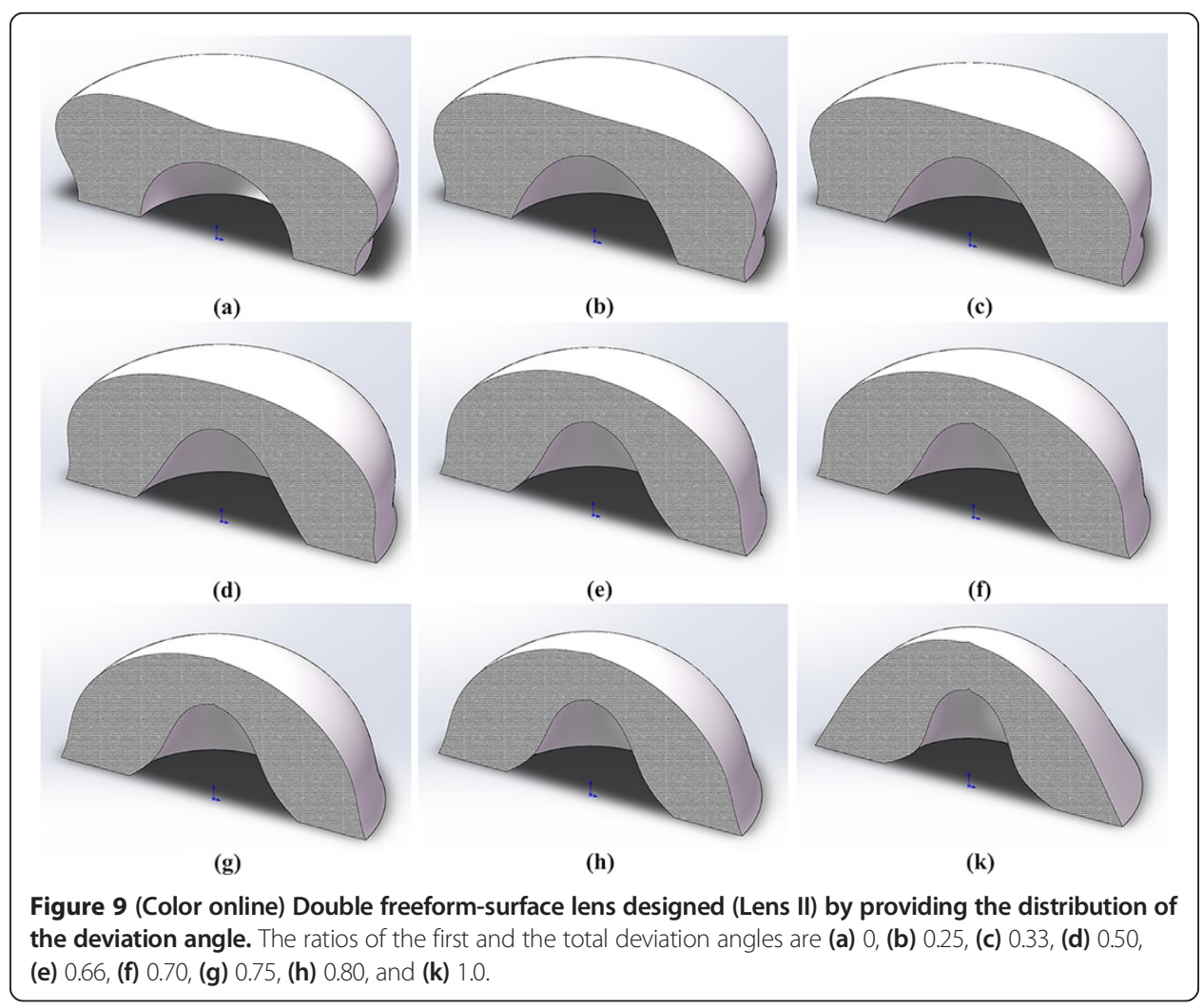


Competing interests

The authors declare that they have no competing interests.

\section{Authors' contributions}

$\mathrm{RH}$ and $\mathrm{XBL}$ contributed in the discussion. $\mathrm{RH}$ drafted the manuscript. $\mathrm{RH}$ and $\mathrm{XBL}$ conceived the idea and revised the manuscript. XBL supervised the project. All authors read and approved the final manuscript.

\section{Acknowledgements}

This work was supported partly by National Science Foundation of China (51376070) and partly by 973 Project of The Ministry of Science and Technology of China (2011CB013105).

Received: 7 October 2013 Accepted: 18 February 2014

Published: 23 April 2014

\section{References}

1. Tonzani S: Time to change the bulb. Nat 2009, 459:312.

2. Hu R, Luo XB, Liu S: Study on the optical properties of conformal coating LED by Monte Carlo simulation. IEEE Photon Technol Lett 2011, 23(22):1673.

3. Hu R, Luo XB, Zheng H: Hotspot location shift in the high-power phosphor-converted white light-emitting diode packages. Jpn J Appl Phys 2012, 51:09MK05.

4. Chi WH, Chou TL, Han CN, Yang SY, Chiang KN: Analysis of thermal and luminous performance of MR-16 LED lighting module. IEEE T Compon Pack T 2010, 33(4):713.

5. Hu R, Yu S, Zou Y, Zheng H, Wang F, Liu S, Luo XB: Near-/mid-field effect of color mixing for single phosphorconverted light-emitting diode package. Photon Technol Lett 2013, 25(3):246-249.

6. Benítez P, Miñano JC, Blen J, Chaves J, Dross O, Hernández M, Falicoff W: Simultaneous multiple surface optical design method in three dimensions. Opt Eng 2004, 43(7):1489-1502.

7. Duerr F, Benítez $P$, Miñano JC, Meuret $Y$, Thienpont $H$ : Analytic design method for optimal imaging: coupling three ray sets using two free-form lens profiles. Opt Express 2012, 20(5):5576-5585.

8. Duerr F, Benítez P, Miñano JC, Meuret $Y$, Thienpont H: Analytic free-form lens design in 3D: coupling three ray sets using two lens surfaces. Opt Express 2012, 20(9):10839-10846.

9. Ding Y, Liu X, Zheng ZR, Gu PF: Freeform LED lens for uniform illumination. Opt Express 2008, 16:12958-12966.

10. Ries H, Muschaweck J: Tailoring freeform lenses for illumination. Proc SPIE 2001, 4442:43-50.

11. Wang L, Qian K, Luo Y: Discontinuous free-form lens design for prescribed irradiance. Appl Opt 2007, 46(18):3716-3723.

12. Wang K, Wu D, Chen F, Liu ZY, Luo XB, Liu S: Angular color uniformity enhancement of white light-emitting diodes integrated with freeform lenses. Opt Lett 2010, 35(11):1860.

13. Chen F, Liu S, Wang K, Qin Z, Liu ZY, Luo XB: Free-form lenses for high illuminance quality light-emitting diode MR16 lamps. Opt Eng 2009, 48(12):123002.

14. Wang K, Chen F, Liu ZY, Luo XB, Liu S: Design of compact freeform lens for application specific light-emitting diode packaging. Opt Express 2010, 18(2):413.

15. Chen F, Wang K, Qin Z, Wu D, Luo XB, Liu S: Design method of high-efficient LED headlamp lens. Opt Express 2010, 18(20):20926.

16. Hu R, Zheng H, Ji CG, Liu S, Luo XB: A method to design freeform lens for uniform illuminance in direct-lit LED backlight with high distance-height ratio. Guilin, China: 13th International Conference on Electronic Packaging Technology and High Density Packaging; 2012:1474-1478.

17. Hu R, Luo XB, Zheng H, Zong Q, Gan ZQ, Wu BL, Liu S: Design of a novel freeform lens for LED uniform illumination and conformal phosphor coating. Opt Express 2012, 20(13):13727.

18. Hu R, Gan ZQ, Luo XB: Design of double freeform-surface lens by distributing the deviation angle for lightemitting diode uniform illumination. Dalian, China: 14th International Conference on Electronic Packaging Technology and High Density Packaging; 2013:1150-1153.

19. Hu R, Gan ZQ, Luo XB, Zheng H, Liu S: Design of double freeform-surface lens for LED uniform illumination with minimum Fresnel losses. Optik 2013, 124:3895-3897.

doi:10.1186/2196-1107-1-3

Cite this article as: Hu and Luo: Adding an extra condition: a general method to design double freeform-surface lens for LED uniform illumination. Journal of Solid State Lighting 2014 1:3. 